15.2;06

\title{
Исследование нелинейных процессов в сегнетоэлектрической керамике в слабых электрических полях
}

\author{
() Н.А. Швецова, С.А. Щербинин, И.А. Швецов, М.А. Луговая, А.Н. Рыбянец \\ Южный федеральный университет, Ростов-на-Дону, Россия \\ E-mail: yfnfif_71@bk.ru
}

Поступило в Редакцию 28 января 2020г.

В окончательной редакции 17 апреля 2020г.

Принято к публикации 17 апреля 2020 г.

\begin{abstract}
Предложен новый метод исследования нелинейных процессов в сегнетоэлектрической керамике при внешних воздействиях, основанный на измерении и анализе пьезорезонансных спектров. Метод обеспечивает высокую точность определения комплексных упругих, диэлектрических и пьезоэлектрических констант пьезоэлектрических материалов, а также их изменений при внешних воздействиях. Для изучения нелинейных процессов выполнены измерения зависимостей комплексных электромеханических параметров „сегнетомягкой“ пьезокерамики на основе цирконата-титаната свинца от постоянного электрического поля. Предложена физическая интерпретация полученных результатов.
\end{abstract}

Ключевые слова: нелинейные процессы, комплексные параметры, сегнетоэлектрическая керамика, метод анализа пьезорезонансных спектров.

DOI: 10.21883/PJTF.2020.14.49666.18224

В последние годы существенно возросли требования к характеристикам ультразвуковых преобразователей для сбора энергии, неразрушающего контроля, медицинской терапии и диагностики. Все большее применение находят новые пьезоэлектрические и композиционные материалы для пьезоэлектрических и ультразвуковых преобразователей с высоким разрешением, чувствительностью и эффективностью [1-3]. Некоторые из этих перспективных материалов характеризуются высокими потерями, и прямое использование стандартных методов определения электрофизических параметров приводит к значительным ошибкам. Основные методы нахождения электрофизических постоянных пьезоэлектрических материалов изложены в стандарте IEEE (1987) [4]. Однако их использование для характеризации новых пьезоэлектрических материалов, таких как пористая керамика, пьезоэлектрические полимеры или композиты, может приводить к значительным ошибкам. Точное описание параметров пьезоэлектрических материалов должно включать оценку диэлектрических, пьезоэлектрических и механических потерь, учитывающих несинфазный отклик материала на входной сигнал. Было предложено множество методов с использованием комплексных констант для учета потерь в материалах с низкой механической добротностью и преодоления ограничений стандарта IEEE [5,6]. Итерационные методы [7-9] обеспечивают возможность точного определения комплексных констант поляризованной пьезокерамики в линейном диапазоне на основе резонансного измерения комплексного импеданса. Метод анализа пьезорезонансных спектров (PRAP) [9] является автоматическим итерационным методом, который может быть использован не только для полной комплексной характеризации пьезоэлектрических материалов, но и для анализа температурных, полевых и временны́х зависимостей комплексных параметров пьезокерамических образцов [10]. Изучение влияния постоянного электрического поля чрезвычайно важно для оценки свойств сегнетоэлектрических материалов, а также исследования релаксационных и доменно-ориентационных процессов $[10,11]$. В настоящей работе для определения комплексных параметров сегнетоэлектрической керамики и исследования нелинейных процессов, происходящих в сегнетопьезоэлектрической керамике под действием постоянного электрического поля, был использован метод анализа пьезорезонансных спектров PRAP [9].

Программа PRAP анализирует импедансные спектры и обеспечивает высокую точность определения полных наборов комплексных упругих, диэлектрических и пьезоэлектрических параметров сегнетопьезоэлектрических материалов, а также их изменений при внешних воздействиях. Эта программа использует обобщенную форму метода Смита для определения свойств материала для любой стандартной резонансной моды и обобщенный метод отношений для радиальной моды [12], справедливый для материалов с произвольной механической добротностью.

Для исследования была выбрана „сегнетомягкая“ пьезокерамика системы цирконата-титаната свинца состава $\mathrm{PbTi}_{0.6} \mathrm{Zr}_{0.336} \mathrm{~W}_{0.006} \mathrm{Mn}_{0.0233} \mathrm{Nb}_{0.0347} \mathrm{O}_{3}$. Исследованная керамика принадлежит области морфотропного фазового перехода и характеризуется низким значением коэрцитивного поля $\left(E_{C} \approx 7.5 \mathrm{kV} / \mathrm{cm}\right)$ и наличием как 90- и 180-градусных доменов, характерных для тетрагональной фазы, так и 71-, 109- и 180-градусных ромбоэдрических доменов [11]. Для экспериментов использовались диски из горячепрессованной пьезокерамики диаметром $6 \mathrm{~mm}$ и толщиной $0.20 \mathrm{~mm}$. Пьезокерамические элемен- 
Комплексные электромеханические параметры пьезокерамики $\mathrm{PbTi}_{0.6} \mathrm{Zr}_{0.336} \mathrm{~W}_{0.006} \mathrm{Mn}_{0.0233} \mathrm{Nb}_{0.0347} \mathrm{O}_{3}$ при нулевом постоянном электрическом поле

\begin{tabular}{c|c|c|c}
\hline \multicolumn{2}{c|}{ Коэффициент электромеханической связи } & \multicolumn{2}{c}{ Диэлектрическая проницаемость } \\
\hline$k_{t}^{\prime}$ & $k_{t}^{/ /}$ & $\varepsilon_{33}^{S /} / \varepsilon_{0}$ & $\varepsilon_{33}^{S / /} / \varepsilon_{0}$ \\
\hline 0.51 & $4.86 \cdot 10^{-5}$ & 242.9 & 11.8 \\
\hline \multicolumn{5}{|c}{ Модули упругости } \\
\hline$C_{33}^{D /}, \mathrm{N} / \mathrm{m}^{2}$ & $C_{33}^{D / /}, \mathrm{N} / \mathrm{m}^{2}$ & $C_{33}^{E /}, \mathrm{N} / \mathrm{m}^{2}$ & $C_{33}^{E / /}, \mathrm{N} / \mathrm{m}^{2}$ \\
\hline $1.80 \cdot 10^{11}$ & $2.16 \cdot 10^{9}$ & $1.32 \cdot 10^{11}$ & $1.60 \cdot 10^{9}$ \\
\hline \multicolumn{5}{|c|}{ Пьезоконстанты } \\
\hline$e_{33}^{\prime}, \mathrm{C} / \mathrm{m}^{2}$ & $e_{33}^{/ /}, \mathrm{C} / \mathrm{m}^{2}$ & $h_{33}^{\prime}, \mathrm{V} \cdot \mathrm{m}$ & $h_{33}^{/ /}, \mathrm{V} \cdot \mathrm{m}$ \\
\hline 10.09 & 0.185 & $4.69 \cdot 10^{9}$ & $1.42 \cdot 10^{8}$
\end{tabular}

ты поляризовались на воздухе путем приложения к серебряным электродам постоянного электрического поля $(\sim 1 \mathrm{kV} / \mathrm{cm})$ при нагревании образца выше температуры Кюри $\left(\sim 340^{\circ} \mathrm{C}\right)$ и охлаждении под действием поля до комнатной температуры.

Анализатор импеданса Agilent 4294A со встроенным модулем постоянного напряжения $( \pm 40 \mathrm{~V})$ использовался для прецизионного измерения пьезорезонансных спектров под действием постоянного электрического поля. При анализе пьезорезонансных спектров использовался модуль сбора данных PRAP для сканирования в постоянном электрическом поле. Время выборки импедансных спектров было равно $3 \mathrm{~s}$.

Комплексные параметры исследуемой пьезоэлектрической керамики, полученные с использованием анализа пьезорезонансных спектров, измеренных до приложения постоянного электрического поля, для толщинной моды колебаний приведены в таблице. Резонансная частота пьезокерамического элемента составляла $11.3 \mathrm{MHz}$. На рис. 1-3 приведены примеры полевых зависимостей электромеханических параметров пьезокерамики. Зависимости носят типичный гистерезисный характер, а наблюдаемые наклоны и асимметрия петель гистерезиса обусловлены исходной поляризацией пьезокерамического элемента.

На рис. 1 приведены зависимости действительной $C_{33}^{D /}$ $(a)$ и мнимой $C_{33}^{D / /}(b)$ частей модуля упругости от постоянного электрического поля $E$, измеренные в области слабых полей, значительно меньших коэрцитивного $\left(E \ll E_{C}\right)$. Положительные значения $E$ соответствуют противоположным направлениям приложенного поля и исходной поляризации пьезокерамики $P$. Частичная деполяризация образца пьезокерамики приводит к уменьшению $C_{33}^{D /}$ и увеличению $C_{33}^{D / /}$ в результате обратимых 71-, 109- и 90-градусных доменных переориентаций, приводящих к уменьшению остаточной поляризации, росту затухания механических колебаний из-за рассеяния на движущихся доменных стенках и системах механиче- ских напряжений и усилению пьезоэлектрического взаимодействия доменов [11]. При отрицательных значениях $E$ поляризация керамики $P$ совпадает с направлением приложенного постоянного поля $E$. Увеличение $C_{33}^{D /}$ и уменьшение $C_{33}^{D / /}$ в этом случае обусловлены дополнительной поляризацией пьезокерамики, вызванной упомянутыми выше процессами доменных переориентаций.

На рис. 2 показаны зависимости действительной $k_{t}^{\prime}(a)$ и мнимой $k_{t}^{/ /}(b)$ частей коэффициента электромеханической связи толщинной моды колебаний от постоянного электрического поля. Частичная деполяризация образца пьезокерамики приводит к уменьшению $k_{t}^{\prime}$ и росту $k_{t}^{/ /}$, вызванным обратимыми 71-, 109- и 90-градусными доменными переориентациями, приводящими к уменьшению остаточной поляризации, росту затухания механических колебаний и усилению пьезоэлектрического взаимодействия доменов. Дополнительная поляризация пьезокерамики при совпадающих направлениях $E$ и $P$ приводит к росту $k_{t}^{\prime}$ и $k_{t}^{/ /}$, вызванному упомянутыми выше доменно-ориентационными процессами.

На рис. 3 приведены зависимости действительной $C_{33}^{E /}(a)$ и мнимой $C_{33}^{E / /}(b)$ частей модуля упругости от постоянного электрического поля. Изменения действительной и мнимой частей модуля упругости $C_{33}^{E}$ определяются рядом конкурирующих факторов, главным образом изменением вклада коэффициента электромеханической связи $k_{t}$ (рис. 2) в соответствии с формулой $C_{33}^{E}=C_{33}^{D}\left(1-k_{t}^{2}\right)$ и изменением модуля упругости $C_{33}^{D}$ (рис. 1).

Наблюдаемый на рис. 1-3 гистерезис полевых зависимостей комплексных электромеханических характеристик „сегнетомягкой“ пьезокерамики обусловлен в основном доменно-ориентационными процессами [13]. Процессы релаксации объемного заряда, отмеченные в [11], в нашем случае являются несущественными из-за использованных величин электрического поля $E$ и времени выборки последовательных импедансных спектров $(\sim 3 \mathrm{~s})$. 

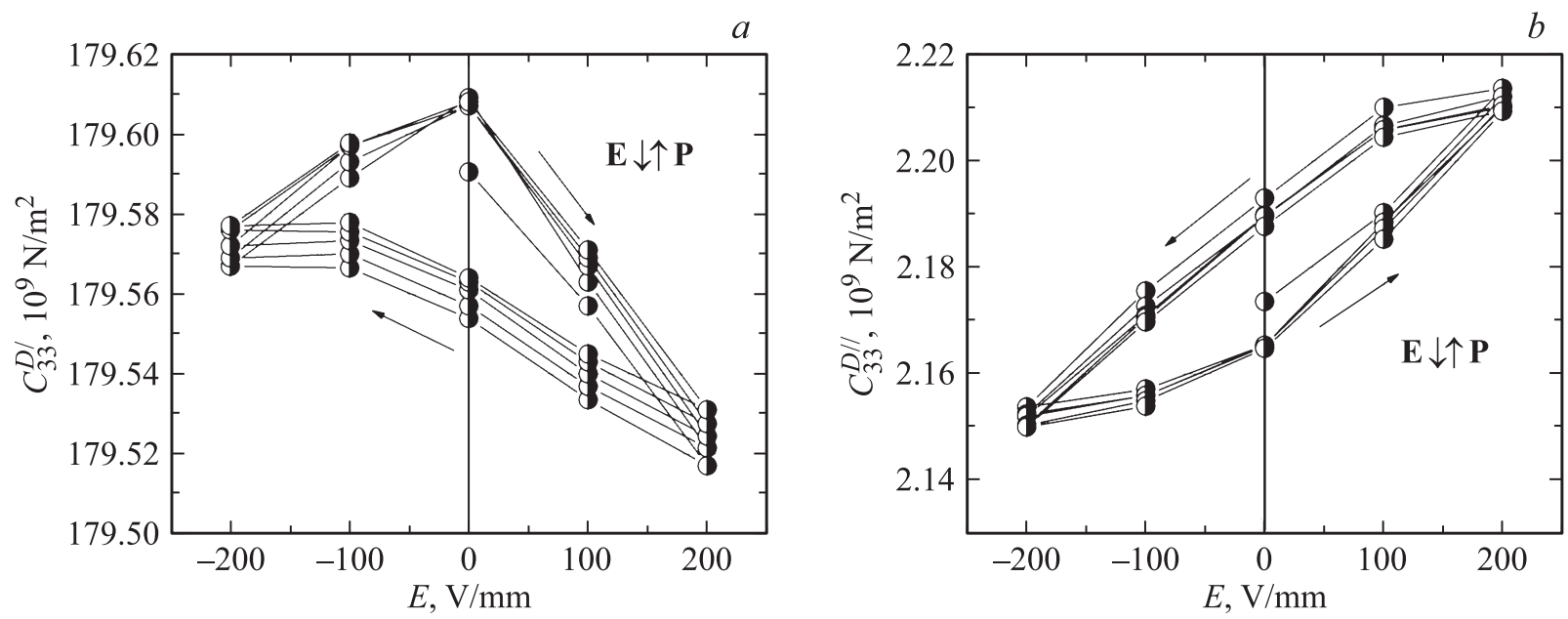

Рис. 1. Экспериментальные зависимости действительной $C_{33}^{D /}(a)$ и мнимой $C_{33}^{D / /}(b)$ частей модуля упругости от постоянного электрического поля для толщинной моды колебаний пьезокерамического диска.
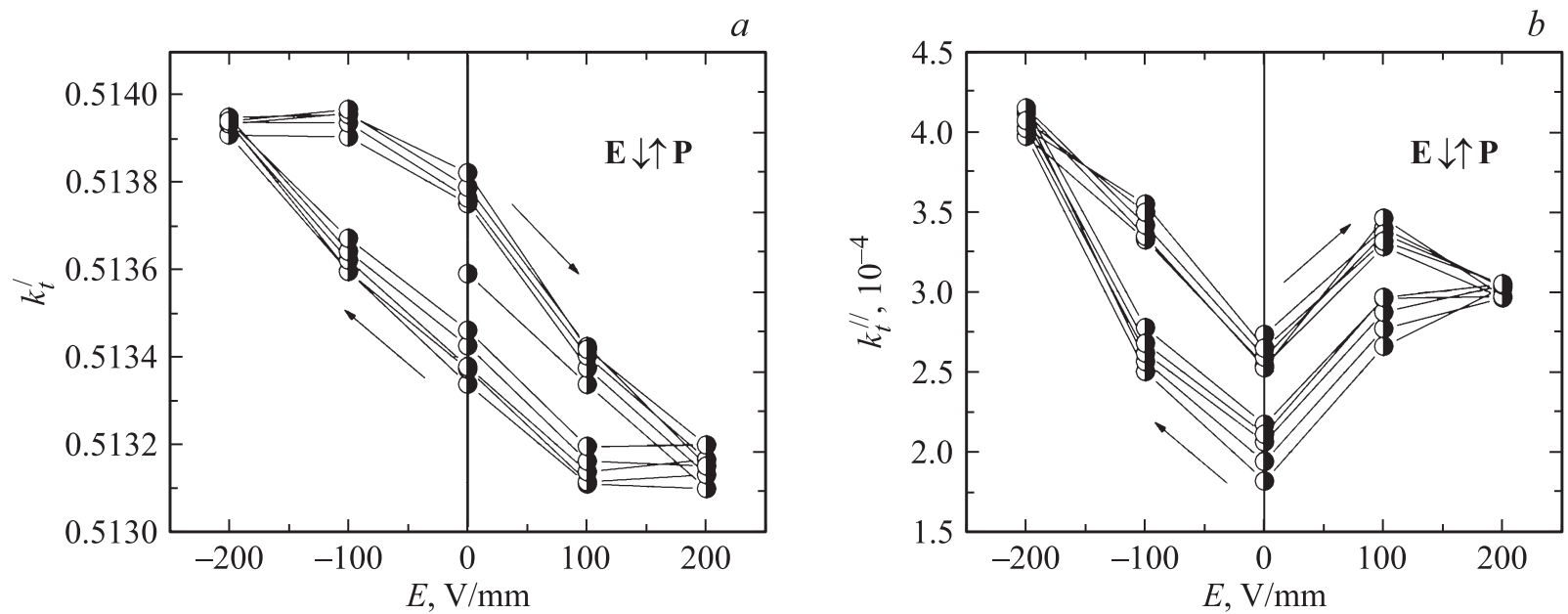

Рис. 2. Экспериментальные зависимости действительной $k_{t}^{\prime}(a)$ и мнимой $k_{t}^{/ /}(b)$ частей коэффициента электромеханической связи от постоянного электрического поля для толщинной моды колебаний пьезокерамического диска.
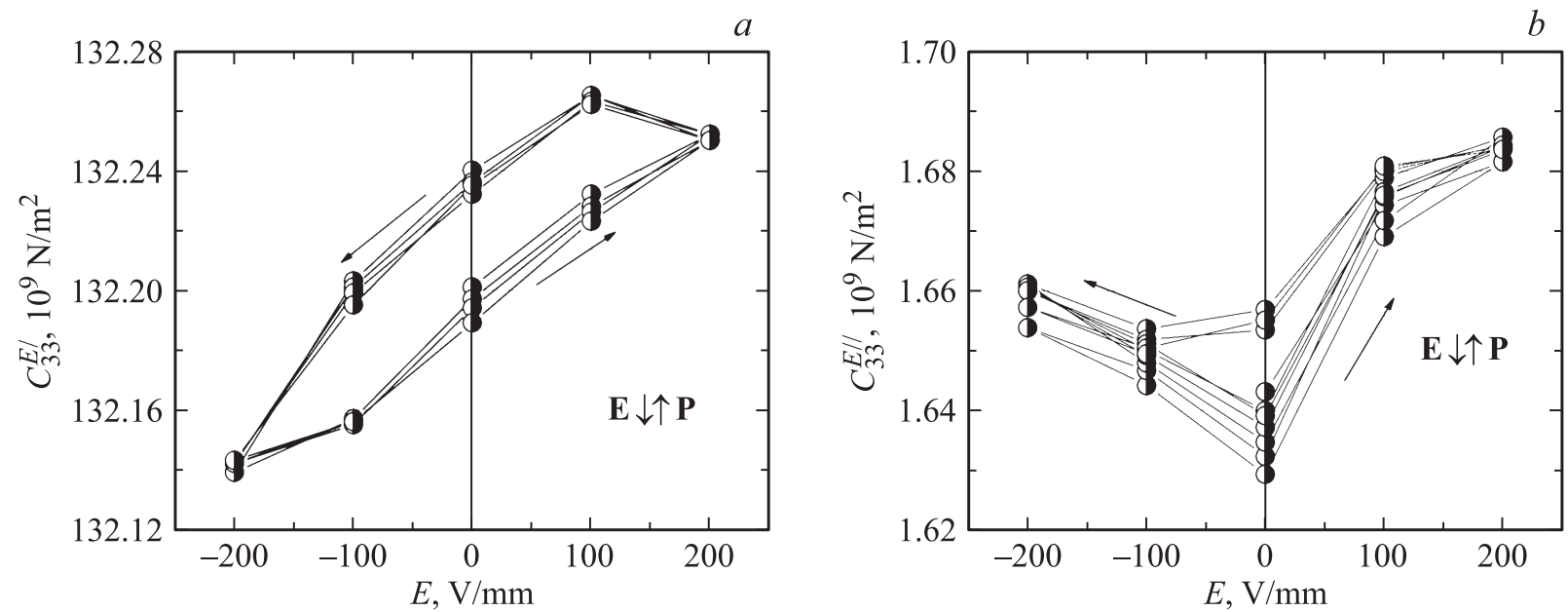

Рис. 3. Экспериментальные зависимости действительной $C_{33}^{E /}(a)$ и мнимой $C_{33}^{E / /}(b)$ частей модуля упругости от постоянного электрического поля для толщинной моды колебаний пьезокерамического диска. 
Разработанный метод комплексной электромеханической характеризации, основанный на анализе пьезорезонансных спектров, позволяет получать полные наборы комплексных упругих, диэлектрических и пьезоэлектрических параметров, а также более точные данные о доменно-ориентационных процессах, происходящих в сегнетоэлектрической керамике при внешних воздействиях

\section{Финансирование работы}

Работа выполнена при финансовой поддержке Министерства науки и высшего образования РФ (государственное задание в сфере научной деятельности, Южный федеральный университет), тема № БАЗ3110/20-3-08ИФ.

\section{Конфликт интересов}

Авторы заявляют, что у них нет конфликта интересов.

\section{Список литературы}

[1] Bowen C.R., Topolov V.Y., Kim H.A. Modern piezoelectric energy-harvesting materials. Springer Ser. in Materials Science. V. 238. N.Y.: Springer, 2016. 152 p.

[2] Rybyanets A.N. // Piezoceramic materials and devices / Ed. I.A. Parinov. N.Y.: Nova Science Publ. Inc., 2010. P. 113-175.

[3] Rybyanets A.N., Naumenko A.A., Lugovaya M.A., Shvetsova N.A. // Ferroelectrics. 2015. V. 484. N 1. P. 95-100. DOI: 10.1080/00150193.2015.1060065

[4] IEEE Standard on piezoelectricity. ANSI/IEEE Std. N.Y.: Institute of Electrical and Electronics Engineers Inc., 1987. $176 \mathrm{p}$.

[5] Rybianets A., Kushkuley L., Eshel Y., Nasedkin A. Accurate evaluation of complex material constants of porous piezoelectric ceramics // Proc. IEEE Ultrasonics Symp. Vancouver: Institute of Electrical and Electronics Engineers, 2006. V. 1. P. 1533-1536. DOI: 10.1109/ULTSYM.2006.389

[6] Kwok K.W., Chan H.L.W., Choy C.L. // IEEE Trans. Ultrason. Ferroelectr. Freq. Control. 1997. V. 44. N 4. P. 733-740. DOI: $10.1109 / 58.655188$

[7] Alguero M., Alemany C., Pardo L., Gonzalez A.M. // J. Am. Ceram. Soc. 2004. V. 87. N 2. P. 209-212. DOI: 10.1111/j.1551-2916.2004.00209.x

[8] Alemany C., Pardo L., Jimenez B., Carmona F., Mendiola J., Gonzalez A.M. // J. Phys. D: Appl. Phys. 1994. V. 27. N 1. P. 148-156. https://doi.org/10.1088/0022-3727/27/1/023

[9] PRAP (Piezoelectric Resonance Analysis Program). TASI Technical Software Inc. [Электронный ресурс]. Режим доступа: www.tasitechnical.com

[10] Esin A.A., Alikin D.O., Turygin A.P., Abramov A.S., Hrescak J., Walker J., Rojac T., Bencan A., Malic B., Kholkin A.L., Shur V.Ya. // J. Appl. Phys. 2017. V. 121. N 7. P. 074101 (1-7). DOI: $10.1063 / 1.4975341$
[11] Shvetsova N.A., Reznitchenko A.N., Shvetsov I.A., Petrova E.I., Rybyanets A.N. Surface acoustic waves method for piezoelectric material characterization // Proc. of the 2015 Int. Conf. „Physics, mechanics of new materials and their applications“. N.Y.: Nova Science Publ. Inc., 2016. P. $415-422$

[12] Smits J.G. // IEEE Trans. Sonics Ultrason. 1976. V. 23. N 6. P. 393-402. DOI: 10.1109/T-SU.1976.30898

[13] Zhao D., Lenz T., Gelinck G.H., Groen P., Damjanovic D., de Leeuw D.M., Katsouras I. // Nature Commun. 2019. V. 10. N 1. P. 2547-2557. DOI: 10.1038/s41467-019-10530-4 